\title{
Genetic Evolution in the Metastatic Progression of Human Pancreatic Cancer Studied by CGH
}

\author{
Gemma Armengol, Gabriel Capellà, Lourdes Farré, Miguel Angel Peinado, \\ Rosa Miró, and Maria Rosa Caballín \\ Unitat d'Antropologia (GA, MRC), Facultat de Ciències, and Unitat de Biologia (RM), Facultat de Medicina, \\ Universitat Autònoma de Barcelona, Barcelona; Laboratori d'Investigació Gastrointestinal (GC, LF), Institut de \\ Recerca Hospital de Sant Pau, Barcelona; Laboratori de Recerca Translacional (GC, MAP), Institut Català \\ d'Oncologia, L'Hospitalet de Llobregat, Barcelona; and Departament de Càncer i Metàstasi (RM), Hospital Duran
} Reynals, L'Hospitalet de Llobregat, Barcelona, Spain

\begin{abstract}
SUMMARY: Metastases are thought to be derived from emerging clones within primary tumors. Although the concept of the clonal evolution of cancer is well defined, the genetic grounds and significance of this process in human cancer progression are still poorly understood. To gain insight into the genetic basis and clonal evolution underlying the metastatic progression of human pancreatic cancer in vivo, we analyzed by comparative genomic hybridization (CGH) chromosomal imbalances in seven metastases originated in nude mice and their three corresponding orthotopically xenografted human pancreatic tumors. All metastases were found to be closely related to the corresponding orthotopic implant, adding many additional changes to the already altered copy number profile of the pancreatic tumors. Recurrent metastasis-specific alterations included gains at 16cen-q22 and 17q21-qter. CGH results from paired specimens strongly suggest that the majority of additional genetic alterations present in metastases are likely to be present in subclones in the primary tumor. (Lab Invest 2001, 81:1703-1707).
\end{abstract}

$D$ isruption of the organization of the genetic material is characteristic of tumor cells. Foulds (1954) and Nowell (1976) hypothesized that genetic instability exists within tumors and that genetic changes (a consequence of the genetic instability) are responsible for cell heterogeneity. Variant cells would emerge throughout the evolution of the tumor, and the rising cell subclones would be subsequently selected according to their biological behavior (vascular invasion, metastatic ability, drug resistance, etc.).

The metastatic ability of tumor cells underlies the most frequent cause of cancer treatment failure. It is generally assumed that metastatic clones arise from variant subclones within the primary tumors. In fact, when analyzing paired primary and metastatic tissues, a progenitor clone may be defined even despite the often-observed heterogeneity (Kuukasjärvi et al, 1997). Controversy exists regarding the kinetics of the heterogeneity in metastatic clones, because it could be caused by an initial diversity of the primary tumor subpopulation or the accumulation of several genetic alterations during the independent evolution of metastasis.

\section{Received August 15, 2001.}

This work was supported by Fondo de Investigaciones Sanitarias (Grant 97/0214) and Plan Nacional I+D (SAF 98-42), and by a grant from the Universitat Autònoma de Barcelona, Bellaterra, Spain.

Address reprint requests to: Gemma Armengol Rosell, Unitat d'Antropologia, Facultat de Ciències, Universitat Autònoma de Barcelona, 08193-Bellaterra, Barcelona, Spain.
Genetic analysis of paired specimens can be informative in revealing subtle differences between primary and metastatic lesions and thereby pinpointing genetic events that could have been critical to metastatic dissemination (Kuukasjärvi et al, 1997). The extent to which the primary and metastatic cell clones are different from one another is an important question for both tumor biology and clinical oncology because this makes it possible to assess the degree of clonal evolution and genetic heterogeneity that characterizes the metastatic progression.

Pancreatic cancer ranks fifth among cancer-related causes of death in Western countries. This type of cancer presents a very poor prognosis because of its metastatic spread and its late diagnosis (Brand and Tempero, 1998). Orthotopic implantation in the corresponding organ of origin in nude mice has proved to be a good in vivo model for the study of this tumor type. A complete concordance is observed between the histological appearance of the primary and the perpetuated tumors in mice (Fu et al, 1992; Reyes et al, 1996), as well as in the expression of tumorassociated antigens ( $\mathrm{Fu}$ et al, 1992). In contrast to subcutaneous implantation, where only local growth occurs, orthotopic implantation reproduces the metastatic behavior of the tumor (Fu et al, 1992; Reyes et al, 1996). Dissemination is present in up to $50 \%$ of perpetuated tumors, is tumor-specific, and keeps stable through a high number of passages (Reyes et al, 1996). The use of solid tumor fragments, where heterogeneity of tumor cell populations is high, in conjunction with the implantation in a favorable microen- 
vironment, may account for this high percentage of dissemination. This model system provides an excellent tool for studying the genetic basis of the metastatic process (Reyes et al, 1996; Tarafa et al, 2000; Villanueva et al, 1998). It is noteworthy that the presence of the host (mouse) desmoplastic reaction does not interfere with the genetic analysis of human xenografted tumors (Hahn et al, 1995; Hilgers and Kern, 1999). Comparative genomic hybridization (CGH) has been established as a powerful approach for carrying out a comprehensive search for gains and losses of DNA sequences in genomic tumor DNA (Kallioniemi et al, 1992). It provides results that are considered to be representative of the entire tumor. Previously, we have shown on eight orthotopic xenografts of pancreatic carcinoma studied by $\mathrm{CGH}$, recurrent gains on chromosomes $8 \mathrm{q}, 15 \mathrm{q}, 16,20 \mathrm{q}$, and 19q, and recurrent losses on chromosomes 18q, 6q, and 9p (Armengol et al, 2000). The copy number karyotype obtained by $\mathrm{CGH}$ is a characteristic genetic fingerprint of a given tumor and has been previously used in the study of progression and clonal evolution between primary tumors and metastases (Gronwald et al 1997; Kuukasjärvi et al 1997). We have used the $\mathrm{CGH}$ technique to address the issue of the origin of genetic divergence in the metastatic process because the dissemination pattern keeps constant through several passages, and distinct passages of orthotopically implanted tumors show a considerable genetic stability (Reyes et al, 1996; Villanueva et al, 1998).

\section{Results and Discussion}

$\mathrm{CGH}$ results are shown in Figure 1. All metastases analyzed had more genetic alterations than the corresponding original tumor. Moreover, in all cases, the metastases had some gains and losses involving the same chromosome regions as the original tumors, but with $\mathrm{CGH}$ profiles deviated more from the balanced value. These quantitative differences could be caused by differences between the extent of the genetic alteration or by a predominance of subpopulations bearing such aberrations (Nishizadi et al, 1997).

\section{Tumor NPg}

Orthotopic xenograft of tumor NP9 at passage \#5 harbored a high number of gains and losses (Fig. 1A). It evolved 6 months after implantation and subsequent serial passaging in the pancreas of five distinct animals. The two metastases analyzed were obtained after 3 months of tumor implantation in the pancreata of nude mice (passage \#1). Therefore, these metastases showed a shorter period of evolution than the orthotopic xenograft. Despite that, all alterations present in the orthotopic implant at passage \#5 were detected in the metastases of passage \#1 as well. Moreover, nonrandom additional alterations were detected in the metastases. Both metastases acquired three de novo alterations, and two of them were shared by both metastases. These observations strongly suggest that both metastases might have arisen from a common clone, already distinct from the still dominant clone in the orthotopic xenograft 6 months later (Fig. 1A).

\section{Tumor NP18}

An orthotopic xenograft of tumor NP18 at passage \#5 and four distinct metastases obtained from passage \#1 were analyzed. The CGH pattern is depicted in Figure 1B. Again, all alterations present in the orthotopic implant were detected in all metastases. Although metastases acquired a high number of gains and losses, these changes were nonrandom, suggesting that they originated from distinct, but related, subclones arising in the orthotopic implant where they were not predominant.

\section{Tumor NP46}

Tumor NP46 showed a moderate tendency to metastasize in the animal model. In this case an orthotopic implant (passage \#6) and a single metastasis from passage \#1 were analyzed. Again, despite the different time elapsed, both the pancreatic implant and the metastasis were closely related, with two additional alterations $(+16 q$ and $+20 q)$ and two quantitative alterations $(+9 p$ and $-18 q)$ being detected in the latter (Fig. 1C).

Several observations arise from the present study. Interestingly, all of the metastases presented the same genetic alterations as the original tumors, as well as new aberrations that may be characteristic of the metastatic process. There was only one aberration with a subtle difference: NP46 presented a loss of whole chromosome 10, whereas the corresponding peritoneal metastasis showed only a loss of $10 \mathrm{q}$. Overall, the total number of aberrations, as measured by $\mathrm{CGH}$, detected in metastases was higher than that observed in orthotopic xenografts, even after a shorter independent evolution. This observation further supports the notion that cancer progression and dissemination associates with the acquisition of further genetic changes (Fearon and Vogelstein, 1990).

In addition, subtle genetic differences between primary and metastatic lesions were revealed. Our data suggest a correlation of metastatic events in pancreatic carcinomas, with an increase in the copy number of genes located at 16q, in particular at 16cen-q22, as well as genes of 17q21-qter, as they appeared in three independent metastases from two distinct tumors. A larger number of paired tumor-metastasis samples should be studied to pinpoint those genetic events predisposing to the metastatic dissemination of pancreatic cancer, as has been performed in other types of tumors (ie, renal clear-cell carcinomas) (Gronwald et al, 1997).

The number of shared genetic changes, as assessed by $\mathrm{CGH}$, has been used as a rough estimate of the degree of clonal relationship between related samples (ie, primary breast tumors and asynchronous metastases) (Kuukasjärvi et al, 1997). Our observations confirm, at the genetic level, that xenografts and 
A

Pancreatic implant \#1

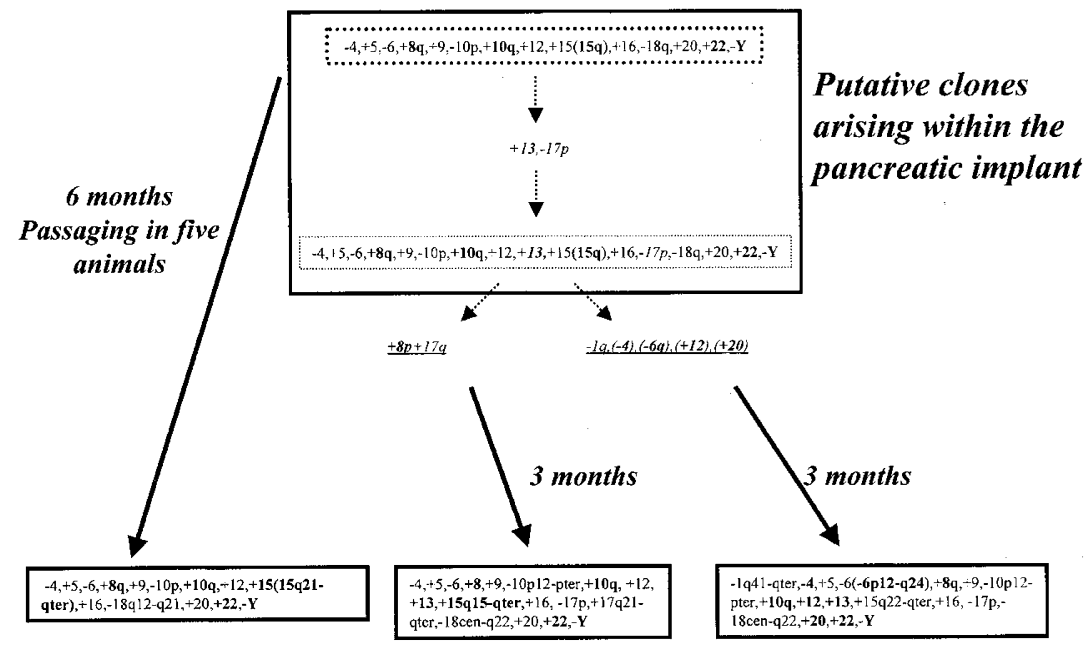

Pancreatic implant \#5

Peritoneal M1 \#1

Peritoneal M1 \#2

B

Pancreatic implant \#1

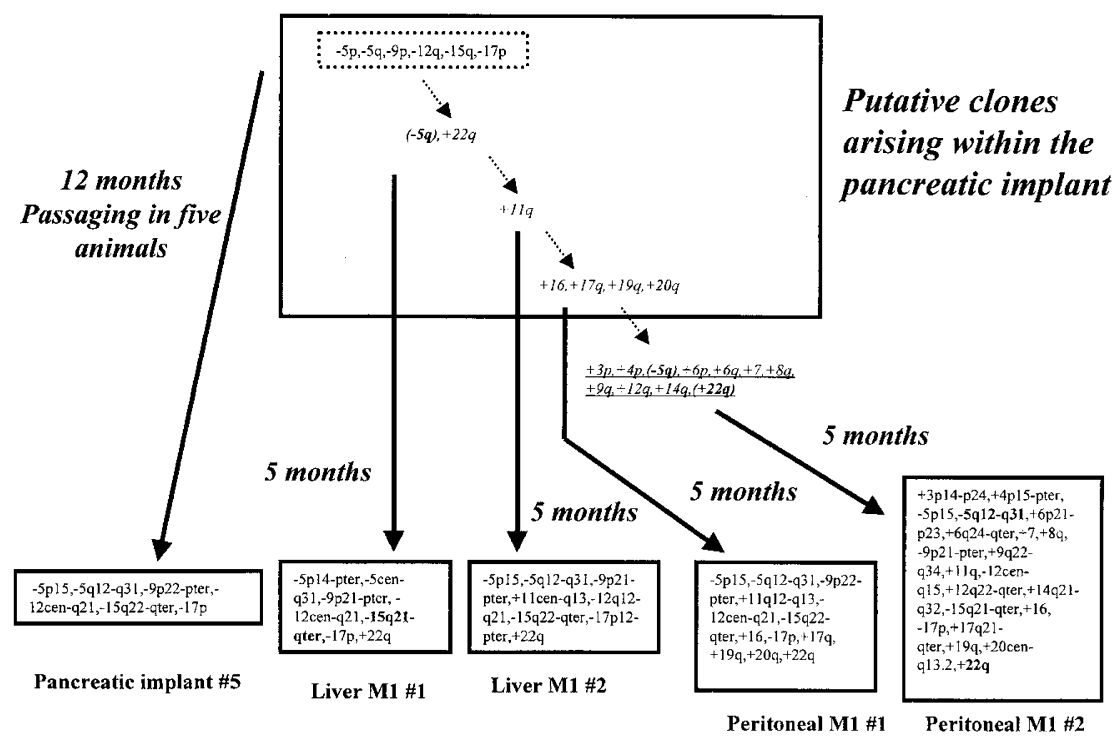

$\mathrm{C}$

Pancreatic implant \#1

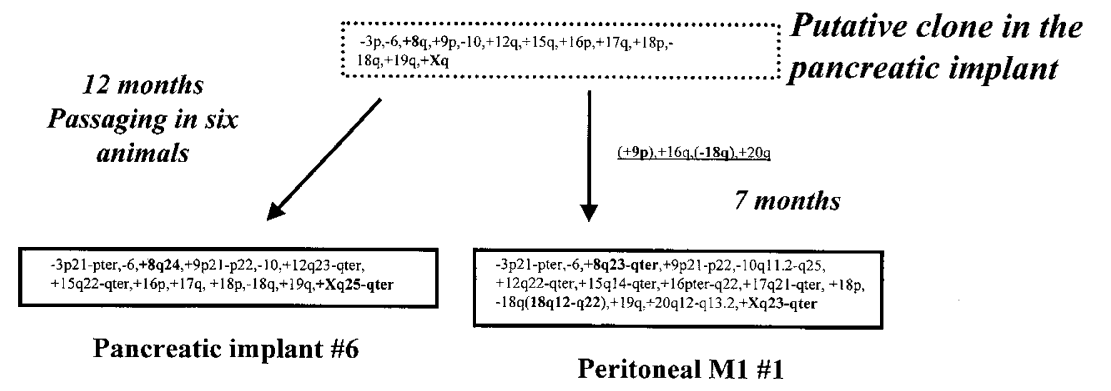

Figure 1.

Hypothetical common stemline and subsequent clonal evolution and tumor progression pathways for xenografted tumors and their metastases in three different cases of pancreatic carcinoma (A, NP9; B, NP18; C, NP46), all of which had metastases clearly clonally related to xenografted tumor. Solid-line boxes depict CGH results and dotted-line boxes show the putative clones present in the xenografted tumor. Subchromosomal breakpoints are not included in the latter. High-level amplifications and homozygous losses are shown in bold, and quantitative differences are given in parentheses (see text). Additional acquired alterations are in italic, and alterations that might have occurred in the metastatic site have been underlined. 
metastases are closely related. Moreover, the use of the orthotopic implantation model has provided us with valuable samples that are of help in elucidating whether additional genetic alterations detected in metastases are already present in subclones within the primary tumor or are acquired after the divergence of the primary and metastatic tumor clones, as postulated by Kuukasjärvi et al (1997) for breast cancer. The presence of additional shared alterations in independent metastases arising simultaneously strongly supports the belief that a significant proportion of the additional genetic alterations is already present in rising subclones within the orthotopic implants.

The evident relationship between a long-time evolved pancreatic implant and the metastases derived from earlier passages reveals that dominant clones within a given microenvironment are important in supporting local growth but may be unrelated to the final outcome of the tumor. Our observations suggest that the intrinsic genetic instability of tumor, evidenced as numerical chromosomal alterations, continuously generates novel subclones that are distinctly selected depending upon the anatomical site: the orthotopic organ (in this case the pancreas) or distal metastases (either liver or peritoneal). Although not dominant in the microenvironment of the orthotopic implant, these subclones can deliver cells into the blood stream where they are more capable of establishing distal metastases. The intrinsic limitation of $\mathrm{CGH}$ and the fact that we have not performed analysis of selected areas within the tumor have precluded the detection of these subclones in defined geographical areas within the tumors.

The acquisition of these novel copy number alterations is probably because of the intrinsic genetic instability of the tumor cells. In recent years, increasing interest has been focused on the existence of chromosomal instability, which is responsible for the existence of a high number of numerical chromosomal alterations in tumors. Our results are consistent with the existence of chromosomal instability in these tumors. Nevertheless, the technique used has not allowed us to know the relative contribution of structural chromosomal alterations to this instability.

Previously, it has been reported that metastases of pancreatic tumors showed a karyotypic complexity similar to that of poorly differentiated tumors (Gorunova et al, 1998). Moreover, Mahlamäki et al (1997) observed that the DNA copy number changes, as assessed by $\mathrm{CGH}$, in 9 metastatic samples did not substantially differ from the overall pattern of imbalances present in 15 primary carcinomas. Furthermore, primary and metastatic pancreatic samples obtained from the same patient displayed almost identical abnormalities. Based on these findings, they concluded that clonal evolution in pancreatic carcinoma cells, at least regarding chromosomal imbalances, was quite similar, irrespective of their location. Discrepancies with our results may be attributed to the use of a model system and/or to distinct sensitivities when using tumor biopsies, where significant "contamination" of nonneoplastic cells (ie, fibroblasts and lym- phocytes) may preclude the detection of some gains or losses.

In conclusion, the ability of CGH to provide detailed comparisons of genetic changes in paired specimens of pancreatic carcinomas and their metastases has enabled the study of the metastatic progression of human pancreatic cancer in vivo. According to our CGH findings, additional genetic alterations present in metastases were probably present in subclones arising within the primary tumor. Some genetic changes, such as gains of $16 q$ and $17 q$, may give clues to the basic molecular mechanisms providing a selective advantage for the metastatic pancreatic process. Although further investigations are required, our results may have implications for understanding the genetic basis underlying the metastatic progression of human cancer in vivo.

\section{Materials and Methods}

We analyzed, by means of $\mathrm{CGH}$, three orthotopic xenografts of human pancreatic carcinomas in nude mice and their seven corresponding metastases obtained from earlier passages. The main characteristics of the orthotopic xenografts are as follows: A NP9 xenograft was derived from a peritoneal metastasis of a poorly differentiated carcinoma and consistently produced peritoneal metastases when allowed to disseminate. A pancreatic implant at passage \#5 (time elapsed between passages \#1 and \#5 was 6 months) and two peritoneal metastases, obtained after allowing dissemination from passage \#1 during 3 months, were studied. A NP18 xenograft was derived from a hepatic metastasis of a poorly differentiated tumor and developed a mixed peritoneal and hepatic dissemination pattern. A pancreatic implant at passage \#5 (time elapsed between passages \#1 and \#5 was 12 months) and a total of four metastases (two hepatic and two peritoneal) obtained after dissemination from passage \#1 were analyzed. Finally, NP46 was originated from a primary pancreatic adenocarcinoma. When allowed to disseminate for 6 months, minor peritoneal metastases were observed. In this case, the pancreatic implant at passage \#6 and one peritoneal metastasis were studied. Results of the three pancreatic xenografts and two metastases were included in a previous study (Armengol et al, 2000).

$\mathrm{CGH}$ analysis was performed as described by Armengol et al (2000). Briefly, tumor DNA was labeled with fluorescein-dUTP (DuPont, Boston, Massachusetts), and normal DNA was labeled with Texas ReddUTP (DuPont) using nick-translation procedures in both. Each of the labeled DNAs (800 ng) were mixed and hybridized to normal metaphase spreads for 2 to 3 days. Hybridizations were analyzed with an Olympus fluorescence microscope and the Cytovision digital image system (Applied Imaging, Sunderland, United Kingdom), which is based on a high-sensitivity integrating monochrome CCD camera and an automated $\mathrm{CGH}$ analysis software package.

Chromosome regions were interpreted as gains when the green-to-red ratio was above 1.25 and as 
losses when the ratio was below 0.75 . A copy number change was considered a high-level amplification when the ratio exceeded 1.5 , and a ratio below 0.5 was considered a homozygous loss or a loss present in almost all of the cell population. These cutoff values were determined based on negative control experiments. In each CGH experiment, a negative control was included. The mean green-to-red ratio in all negative $\mathrm{CGH}$ experiments remained between 0.75 and 1.25 .

\section{References}

Armengol G, Knuutila S, Lluís F, Capellà G, Miró R, and Caballín MR (2000). DNA copy number changes and evaluation of MYC, IGF1R, and FES amplification in xenografts of pancreatic adenocarcinoma. Cancer Genet Cytogenet 116: 133-141.

Brand RE and Tempero MA (1998). Pancreatic cancer. Curr Opin Oncol 10:362-366.

Fearon ER and Vogelstein B (1990). A genetic model for colorectal tumorigenesis. Cell 61:759-767.

Foulds L (1954). The experimental study of tumor progression: A review. Cancer Res 14:327-339.

Fu X, Guadagni F, and Hoffman RM (1992). A metastatic nude-mouse model of human pancreatic cancer constructed orthotopically with histologically intact patient specimens. Proc Natl Acad Sci USA 89:5645-5649.

Gorunova L, Höglund M, Andrén-Sandberg A, Dawiskiba S, Jin Y, Mitelman F, and Johansson B (1998). Cytogenetic analysis of pancreatic carcinomas: Intratumor heterogeneity and nonrandom pattern of chromosome aberrations. Genes Chromosom Cancer 23:81-99.

Gronwald J, Storkel S, Holtgreve-Grev H, Hadaczek P, Brinkschmidt C, Jauch A, Lubinski J, and Cremer T (1997). Comparison of DNA gains and losses in primary renal clear cell carcinomas and metastatic sites importance of $1 q$ and $3 p$ copy number changes in metastatic events. Cancer Res $57: 481-487$.

Hahn SA, Seymour AB, Shamsul Hoque ATM, Schutte M, da Costa LT, Redston MS, Caldas C, Weinstein CL, Fischer A, Yeo CJ, Hruban RH, and Kern SE (1995). Allelotype of pancreatic adenocarcinoma using xenograft enrichment. Cancer Res 55:4670-4675.
Hilgers W and Kern SE (1999). Molecular genetic basis of pancreatic adenocarcinoma. Genes Chromosom Cancer 26: 1-12.

Kallioniemi A, Kallioniemi O-P, Sudar D, Rutovitz D, Gray JW, Waldman F, and Pinkel D (1992). Comparative genomic hybridization for molecular cytogenetic analysis of solid tumors. Science 258:818-821.

Kuukasjärvi T, Karhu R, Tanner M, Kahkonen M, Schaffer A, Nupponen N, Pennanen S, Kallioniemi A, Kallioniemi O-P, and Isola J (1997). Genetic heterogeneity and clonal evolution underlying development of asynchronous metastasis in human breast cancer. Cancer Res 57:1597-1604.

Mahlamäki EH, Hoglund M, Gorunova L, Karhu R, Dawiskiba $\mathrm{S}$, Andren-Sandberg A, Kallioniemi O-P, and Johansson B (1997). Comparative genomic hybridization reveals frequent gains of $20 q, 8 q, 11 q, 12 p$, and $17 q$, and losses of $18 q, 9 p$, and $15 q$ in pancreatic cancer. Genes Chromosom Cancer 20:383-391.

Nishizadi T, DeVries S, Chew K, Goodson III WH, Ljung B-M, Thor A, and Waldman FM (1997). Genetic alterations in primary breast cancers and their metastases direct comparison using modified comparative genomic hybridization. Genes Chromosom Cancer 19:267-272.

Nowell PC (1976). The clonal evolution of tumor cell populations. Science 194:23-28.

Reyes G, Villanueva A, García C, Sancho FJ, Piulats J, Lluís $F$, and Capellà G (1996). Orthotopic xenografts of human pancreatic carcinomas acquire additional genetic aberrations during dissemination in nude mice. Cancer Res 56:57135719 .

Tarafa G, Villanueva A, Farré L, Rodriguez J, Musulén E, Reyes G, Seminago R, Olmedo E, Paulés AB, Peinado MA, Bachs O, and Capellá G (2000). DCC and Smad4 alterations in human colorectal and pancreatic tumour dissemination. Oncogene 19:546-555.

Villanueva A, García C, Paulés $A B$, Vicente $M$, Megías $M$, Reyes G, de Villalonga P, Agell N, Lluís F, Bachs O, and Capellá G (1998). Disruption of the antiproliferative TGF-beta signaling pathways in human pancreatic cancer cells. Oncogene 17:1969-1978. 